\title{
Complementary Roles of Software-based Scaffolding and Teacher-Student Interactions in Inquiry Learning
}

\author{
Iris Tabak and Brian J. Reiser
}

Northwestern University

\begin{abstract}
Working in small groups with computer-based learning environments provides an opportunity for students to investigate and discuss their own explanations of natural phenomena. Incorporating domain-specific strategic support in the design of these environments can make student investigations and discussions more productive by focusing them on key variables and relationships in the domain. However, interacting with these environments may not be enough to help students develop understandings and ways of communicating that are consistent with scientific views. A support system that combines interactions with these environments with teacher-student discussions in both small-group and whole-class formats provides more comprehensive support.

We describe a computer-based investigation environment that incorporates domain-specific strategic support, where students investigate an episode of natural selection in the wild. The environment was used as part of a unit on evolution at a Chicago public high school. We illustrate the complementary roles of this environment and studentteacher discussions in both small-group and wholeclass formats in supporting science learning.
\end{abstract}

Keywords - classroom interaction, science education, computer-based inquiry support

\section{Introduction}

Learning science consists of developing ways of examining and explaining phenomena that are consistent with the practices of the scientific community. Definitions of science learning that emphasize developing cognitive structures (Glynn \& Duit, 1995; White, 1993), and that emphasize developing particular ways of communicating (Lemke, 1990; Pea, 1991), both call for learning to occur in a context where students actively engage in designing experiments, making observations, and constructing, communicating and debating explanations. One successful approach for creating such a context is having students conduct investigations in small collaborative work groups (Minstrell \& Stimpson, 1996), often using computerbased learning environments to design and run experiments (Roschelle, 1992; Roth, 1995). This approach provides students with the opportunity to spend a considerable amount of time engaging in the process of science.

The degree to which the student talk and understandings that emerge from these collaborations converge with scientific views depends on the types of interactions students have with the environment, and the types of discussions that evolve around these interactions. We suggest that these interactions and discussions can be more profitable if the studentdirected inquiry takes place in investigation environments employing domain-specific support that is designed to focus students on the key dimensions, relationships and processes in a domain. In addition, it is important to combine student collaborations using these environments with teacher-student interactions in small-group and whole-class forums. Teachers can model profitable investigation strategies when talking with students during their investigations, and they can help them articulate their findings in ways that are more consistent with scientific views. Whole-class discussions can supplement the support provided by teacher-student interactions in small-groups by sharing insights and guidance across groups.

Earlier research has tended to focus on the role of computer-based learning environments in facilitating discussions (Pea, 1991; Roth, 1995), or on the interrelationships between teacher direction in smallgroup and whole-class discussions (Minstrell \& Stimpson, 1996). Our goal is to explore how to design an integrated unit that capitalizes on the strengths of all three types of support: computerbased scaffolding, teacher-student interactions during small-group work, and teacher-student interactions during whole-class discussions. Understanding how the design of learning environments guides students' inquiry decisions and the content of their discussions, and how combining different discussion formats can 
help students realize more learning opportunities than any format alone, can help us design more effective contexts for learning through computer supported collaborations.

In this paper we discuss our approach to combining these three types of support. The research we present is part of a broader project, BGuILE (Biology Guided Inquiry Learning Environments), studying ways to support learning through studentdirected inquiry in high school biology classes. We describe an environment we designed incorporating domain-specific support in which students can investigate a natural selection event. This environment was used as part of a unit on evolution in an introductory biology class at a Chicago public high school. We illustrate, through small-group and whole-class discussion episodes from this class, how the computer environment and discussions fulfill complementary roles in fostering scientific ways of examining and explaining natural selection phenomena.

\section{Complementary Supports for Science Learning}

\subsection{Domain-specific inquiry support}

Our approach to computer-based inquiry guidance that we call domain-specific strategic support makes explicit the implicit strategies used by experts during investigations. In developing this type of scaffolding we consult with domain experts, analyze scientific reports and studies of students' conceptions in order to derive an investigation model (Tabak, Smith, Sandoval, \& Reiser, 1996) . An investigation model identifies the set of variable, comparison and relationship categories that experts use when conducting investigations in the domain.

Implementing the domain-specific strategic support involves making the components of the investigation model objects that can be manipulated in the software.

For example, the strategy "compare variation in physical characteristics between animals that die and animals that survive" which is part of an investigation model for natural selection, would be an action students can perform in the computer environment by making a menu selection or other interface manipulation. This guides data generation and interpretation by focusing students on key variables and encouraging particular comparisons. In contrast, domain-general environments support data query through selecting data manipulation actions, such as selecting a frequency count, or data representation objects, such as selecting a histogram. In this type of environment comparison categories are left implicit. For example, comparing histograms of data from two time periods or histograms of data from two populations are represented as performing the same action, although conceptually they represent two different actions with very different knowledge goals. Explicitly distinguishing between comparison types can help students learn to associate particular comparisons with particular inquiry goals.

We designed an environment incorporating domain-specific strategic support, called the Finch Scenario, to help students understand the process of natural selection and the ways in which scientists investigate and construe natural phenomena. This scenario is based on a longitudinal study of finches in the Galápagos (Grant, 1986). In this environment students are asked to explain why so many finches in a population of finches on a Galápagos island are dying, and more importantly, what enables the surviving finches to survive during a crisis period in 1977. Students may take quantitative measurements of environmental factors (e.g., amount of rainfall), and of the distribution of various structural characteristics of the birds (e.g., weight, beak length). Students can also examine field notes collected about the plants, animals, and environment of the island. The field notes provide students with important behavioral information. The environment includes data spanning 8 time periods representing dry and wet seasons during a baseline, non-crisis year, during crisis years, and during a post-crisis year.

\subsubsection{Data generation and interpretation: Question types}

The ways the system enables students to generate and observe data are designed to draw their attention to important questions and comparisons in the domain. Data requests are made through a question-based interface, where students select a question type and a question stem to construct a complete question. Question types represent comparison categories and question stems represent variable categories based on an investigation model for natural selection. An example completed question is "Are there changes between time periods in the variation of structural traits? (see Figure 1)." 


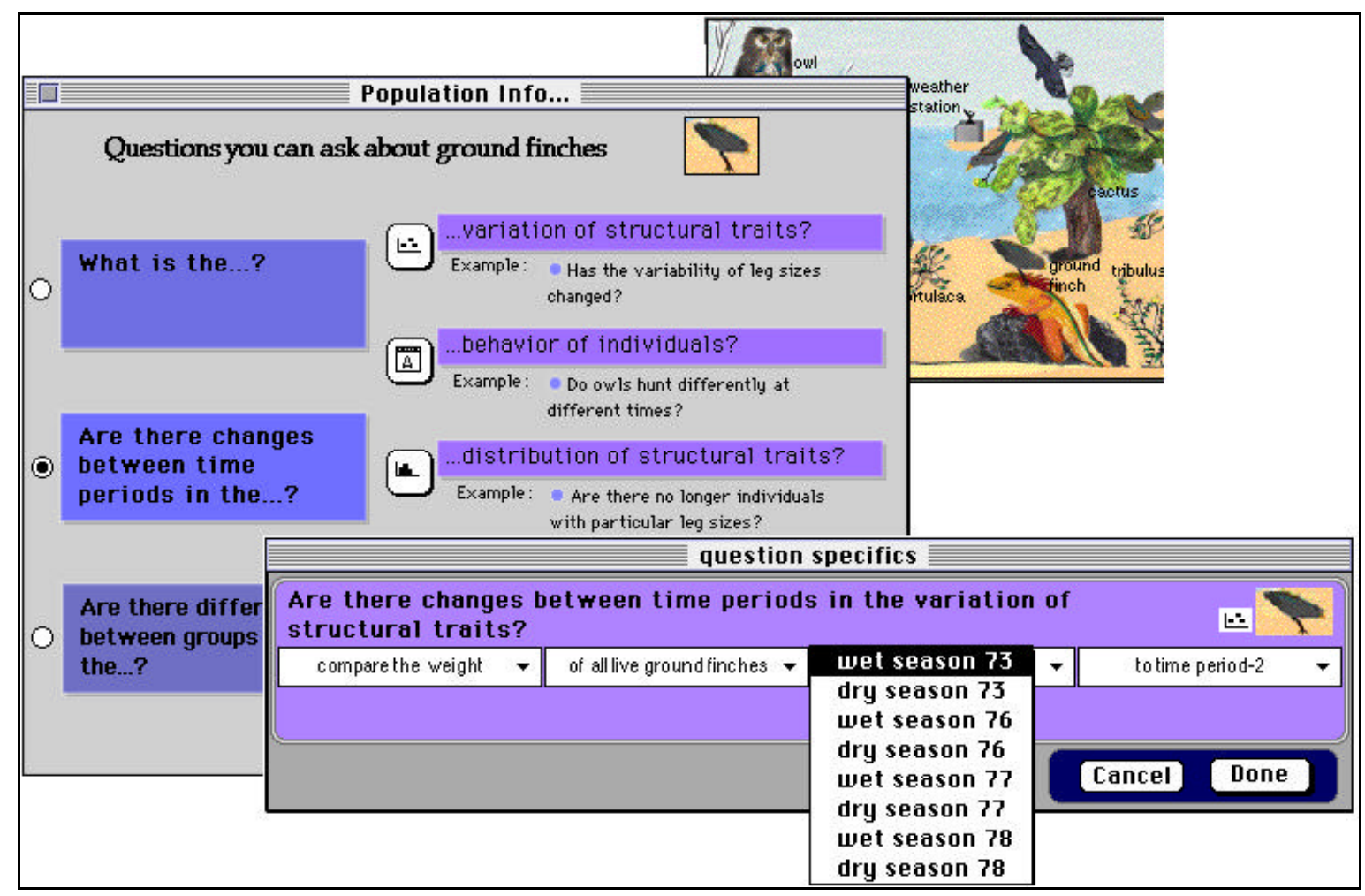

Figure 1. The questions interface and dialogue resulting from selecting "Are there changes between time periods in the..." - "...variation of structural traits?" In the dialogue students specify structure, group, and two time periods.

Questions students can ask include the following:

- Are there changes between time periods in the behavior of individuals? - This can help to determine whether a selective pressure is acting on this population.

- Are there differences between groups in the variation of structural traits? - This question displays individual values on a target trait for two separate populations or subgroups. For example, students could compare weight or size of living and dead finches. This analysis also allows students to access individual finch profiles from the individual data points to explore individual differences that account for these trends.

- Are there changes between time periods in the distribution of structural traits? - This displays separate histograms of the values on a trait in a population for the chosen time periods. This can help to determine whether a natural selection event is occurring, because by definition natural selection results in an increased proportion of the selected trait in the population.
Students can make comparisons of aggregate data, such as differences in the beak length of live and dead birds in the dry season of 1977, and directly access profiles for individual birds through graphs generated from these comparisons. Profiles are cross-referenced to field notes showing behavioral descriptions of finches, such as descriptions of finches' foraging and mating behaviors (see Figure 2).

Students also have the ability to annotate data (see the white scrolling rectangular region in the graph and field note windows in Figure 2). This provides opportunities for students to reflect on and interpret results as the investigation progresses. These annotations are not required, however, because we want students to be able to explore the environment and discover interesting data that requires explanation, rather than forcing them to articulate an hypothesis prior to every access of data.

\subsubsection{Data organization: Evidence categories}

The Finch Scenario includes a data log that helps students manage the complexity of the investigation by organizing the data they collect according to evidence categories, provided by the system, that are pertinent to investigating natural selection (see Figure 

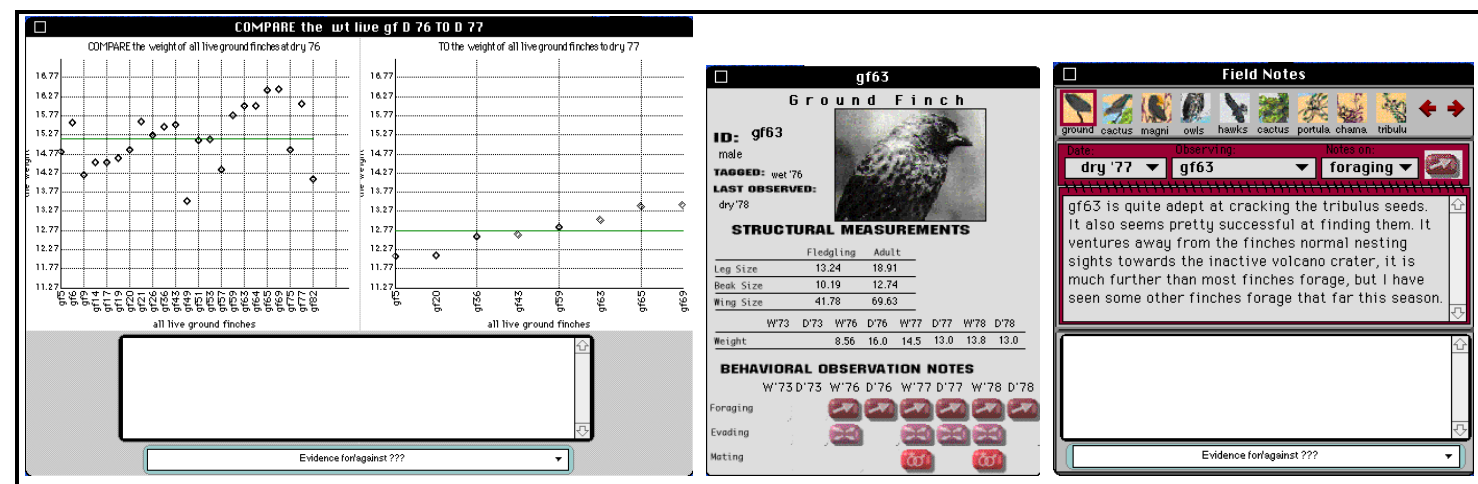

Figure 2: These screens show the graph resulting from the previous dialogue selection of "compare weight of all live ground finches between dry season ' 76 and dry season '77." Clicking on individual data points in the graphs brings up an individual's profile, and profiles are linked to observations of that individual in the field notes window.

3). Any observation that students make is automatically stored in the data log under the "unsorted" category (last row in the data log window in Figure 3). Students can categorize a piece of data either in the data log directly by dragging the thumbnail of the data into the desired slot (row), or when viewing the data using a pull down menu listing each of the categories (shown in Figure 2, it is the wide button with a pop-up arrow at the bottom of the graph window and the field notes window labeled "Evidence for/against ???"). Students working on the Finch Scenario collect between 30 and 60 pieces of data in the process of constructing a causal story. Sorting the data by evidence categories makes this task more manageable by enabling students to attend to subsets of the data. Deciding how to categorize a particular piece of evidence encourages students to reflect on the relationship of individual data to the overall goal of the investigation.

The domain-specific strategic support in the Finch Scenario suggests inquiry actions that students can take, and helps students identify issues that are important to consider when reasoning about natural selection. For example, the question-based interface helps students identify differences in structure between animals that survive and animals that die as an important dimension to consider when reasoning about this domain. Students interacting with the Finch Scenario need to decide which comparison to choose, what variable to observe, and how to categorize their data. The domain-specific strategic support makes it more likely that their discussions when making these choices will center on the important conceptual objects of the domain, because these objects are the options that students can choose. Students will discuss whether they should observe the variation of leg length or beak length in the population, and whether a change in distribution can be noted. Variation and changing distributions are central principles in the process of natural selection, and therefore, this type of support drives students' discussions to converge with scientific ways of examining natural selection.

\subsection{Combining small-group and whole- class discussions}

The Finch Scenario is designed to focus students on salient principles of the domain, and on particular ways of thinking about the domain. However,

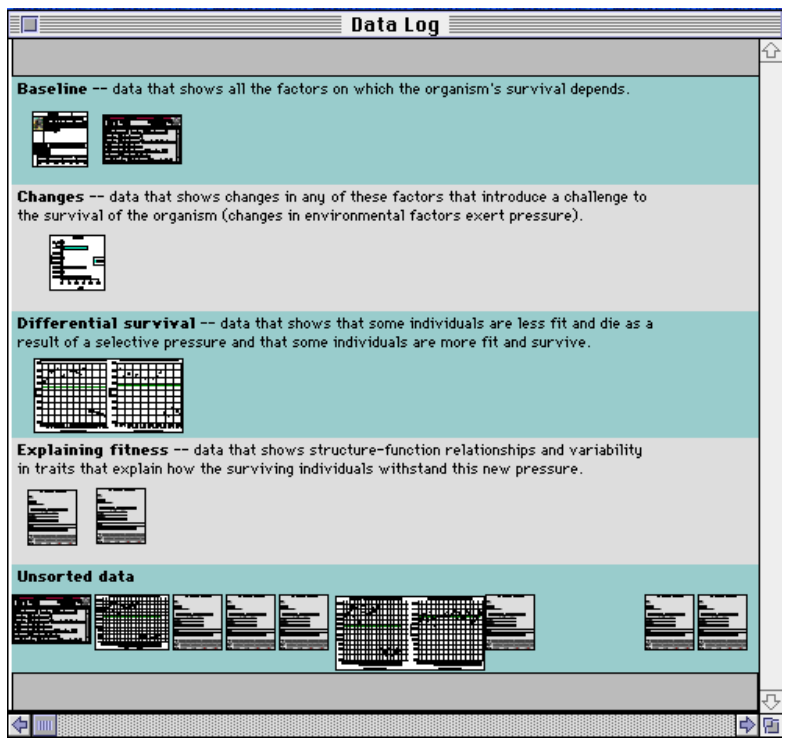

Figure 3. This screen shows the data $\log$ where all student observations are stored. Students can organize their data according to data categories that are pertinent to investigating natural selection. 
students may not reflect on their interactions with the activity and therefore may not realize these opportunities for learning (Shute, Glaser, \& Raghavan, 1989) . Further, students may interpret data in ways that are not consistent with scientific views. Teachers can encourage students to attend to particular features of the environment, to reflect on their findings, and help students describe their conclusions in the language of science through conversations during small-group investigations (Roth, 1995). In this way, teacher-student discussions during small-group investigations can augment the domain-specific strategic support provided by the computer-based learning environment. These teacherstudent discussions, however, also have limitations that can be compensated for by interspersing wholeclass discussions with the small-group investigations, as described in the two sections below.

\subsubsection{Private versus public learning opportunities}

A typical classroom may consist of six to ten work groups. This relatively large number of groups constrains the amount of time the teacher can spend with any one group, therefore constraining the extent of modeling and coaching that the teacher can provide. Further, the benefit that a group receives from interacting with the teacher may depend on the investigation path they have explored so far, or on the particular juncture of their investigation at the time of interaction. However, it is difficult to plan the appropriate points at which the teacher should visit each group, and in many cases the point at which the teacher visits a group is arbitrary. This suggests that some groups may experience richer exchanges with the teacher. Different groups' interactions with the computer-based learning environment may result in very different investigation paths, experiences and interpretations (Roschelle, 1992). As a result some groups may realize learning opportunities that other groups never encounter. Whole class discussions can make these "private" learning opportunities "public" by sharing ideas, questions, insights, and guidance that came up in individual small-group discussions (Minstrell \& Stimpson, 1996) .

\subsubsection{Local versus global constructions}

Students conducting an investigation are focused on interpreting individual pieces of data and trying to relate these isolated observations to construct a causal story for the problem phenomenon. As a result, student-student and student-teacher interactions at the computer tend to focus on these more local interpretations and understandings. Students debate what observations to make, and teachers help students articulate their findings, but little or no attention is directed toward understanding how these findings are representative of broader domain theories or principles. Yet, one of the main goals of learning science through student-directed inquiry is to develop an understanding of domain theories by investigating particular cases. These goals are better achieved by interleaving investigation sessions with whole-class discussion devoted specifically to reflecting on the strategies used in the investigations and on generalizing understandings from the problem phenomenon.

\section{Classroom enactment}

We used the Finch Scenario software as part of a six week unit on evolution in two Chicago area public high schools during the 1996-1997 school year. In this paper we describe interactions from a regular level introductory freshmen biology class in one Chicago public high school. The unit includes two computer-based investigations, as well as a number of paper-based staging activities and discussions that lead up to the more complex computer-based investigations. Our unit design interleaved investigation sessions in small collaborative groups with whole class discussions where students reported on and discussed the history of their investigations, strategy use and findings. Throughout the investigation sessions the teacher (who was audio recorded) went from group to group answering questions, and coaching students through the process of inquiry. In implementing this curricular design we are investigating how to use teacher-student interactions in small-group and whole-class discussions to help students engage in productive investigations, and reflect on and synthesize what they have learned following their investigations.

In this paper we examine whether this curricular design was successful in eliciting the types of discussions we set as a goal. In future papers we will report on the learning outcomes of these discussions based primarily on pre- and post- tests and interviews that we conducted eliciting students' understanding of natural selection. The following examples are discussion episodes from our classroom study that illustrate the complementary roles of the domainspecific strategic support, small-group and wholeclass discussions.

\subsection{Guiding student inquiry through domain-specific strategic support}

Students conducted their investigations in groups of three or four. The activity extended over seven 50 minute class periods, and included a total of 4 class periods devoted to investigation sessions. In these investigations each group collected between 30 and 60 observations, and constructed one or two possible explanations. We taped one group throughout the activity (selected randomly from the students volunteered to be interviewed before and after the unit), and conducted more general observations of the 
remaining groups. Although there is some variability among groups in their interaction styles, the group we taped is fairly representative.

The three students in this group, two girls and a boy, discussed what observations to make, and debated interpretations before making data annotations. One person operated the mouse and keyboard throughout the class session, but students sometimes changed roles between class periods. The two girls were engaged in the activity fairly consistently, while the boy was more likely to become distracted. Interestingly, in other groups there was more variability in engagement patterns and each of the group members tended to be engaged or less engaged at different times.

The following episode is from the students' second investigation session. It demonstrates how the students are supported in making decisions on what observations to make and how the observations are related to the overall goal of the investigation through the questions-based interface and the data log. It also shows how students draw on their earlier investigation experiences in the class, and on each other to help form their decisions and interpretations.

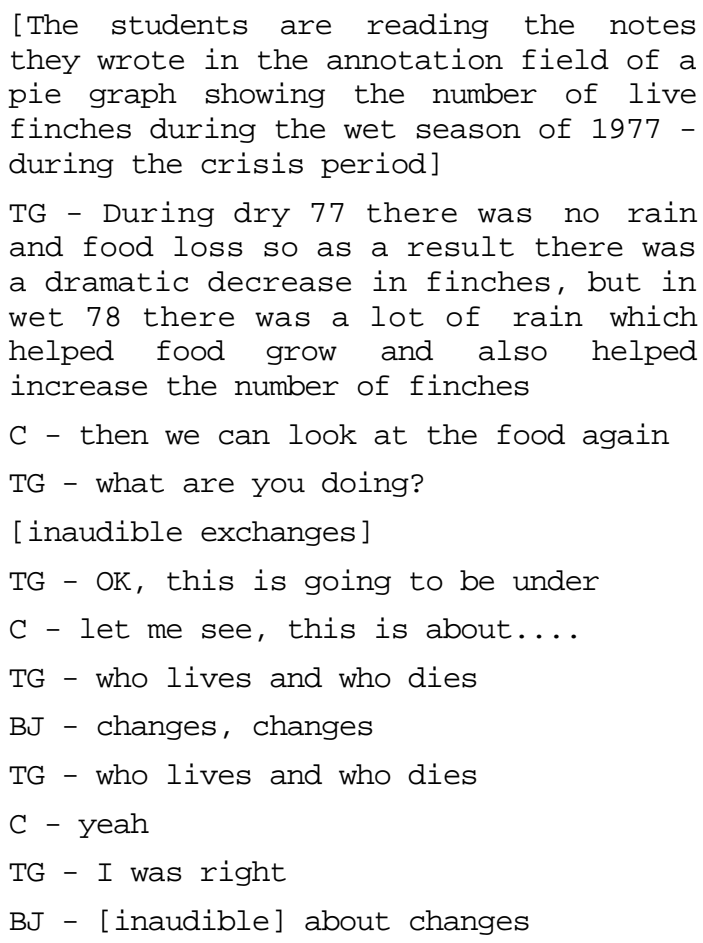

In the quote above, students use the annotation facility to write down a conclusion they reached regarding the relationship between rainfall, food availability and finch mortality. They view the options listed under a menu depicting the category options they can choose from to categorize this piece of data. The group deliberates between two categories,
Changes and Differential Survival, considering how this observation relates to the overall goal of the investigation, something they may not have done spontaneously. Unfortunately, the students do not articulate their justifications for the different category decisions which would have made it clearer to their partners and possibly to themselves why they supported one category over the other.

After categorizing their observation the students go back to the question-based interface to ask a new question:

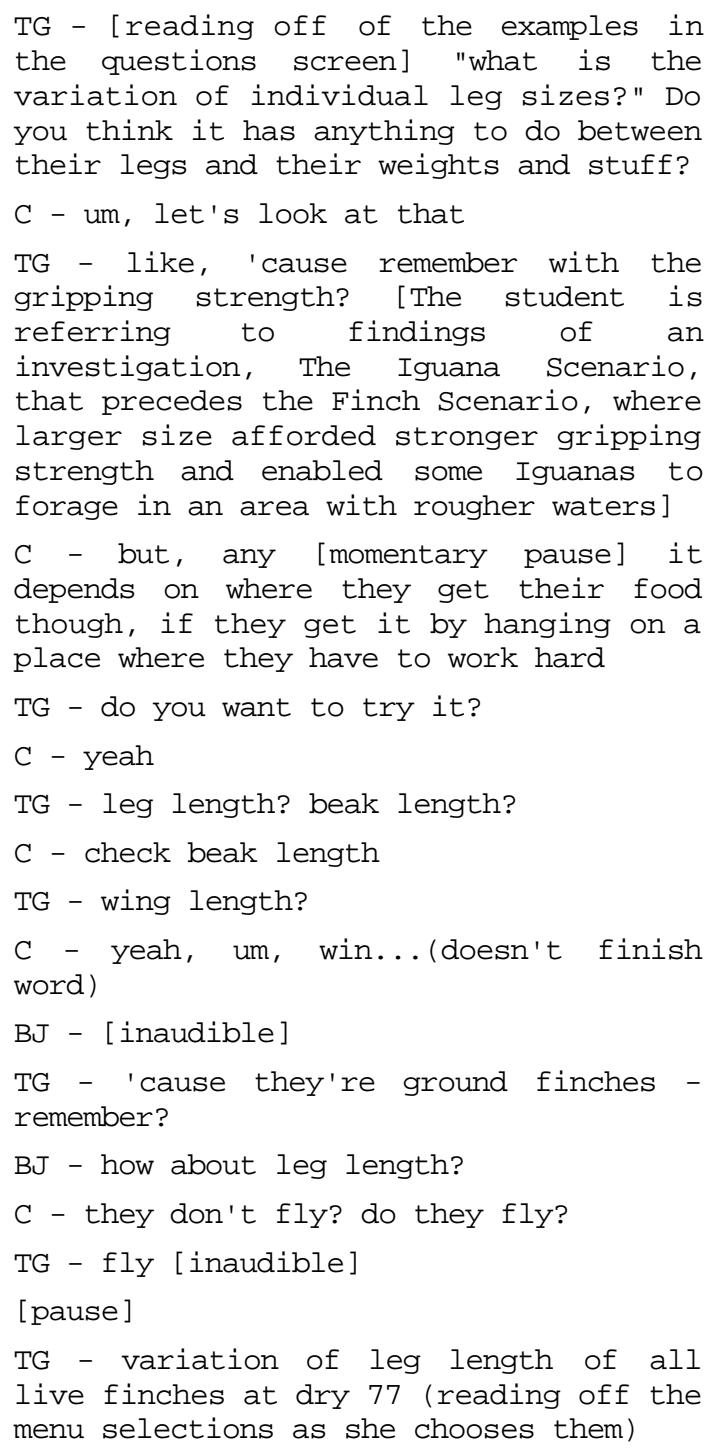

The students employ a bottom-up-top-down approach, initially, they simply read the options that they can select and use that as a prompt for considering whether physical characteristics are relevant variables to examine. At this point, they stop and reflect, in a more theory driven approach, using their findings from an earlier investigation to evaluate whether they 
should make this observation. Once they decide to make this type of observation, they again are planful rather than arbitrary in choosing the specific physical characteristic to observe.

The students deliberate between observing beak length, leg length and wing length. It is clear from the dialogue that they decide against selecting wing length, because they have determined that ground finches do not fly and therefore that would be an irrelevant observation. It is less clear why they decide to observe leg length rather than beak length, although it is likely that this is because they are influenced by an earlier investigation (they make reference to it in the dialogue) where leg size was an important factor.

The structure of the interface in this case seems effective in suggesting relevant observation categories. However it was flexible enough that it was not overly constraining, creating a need for making decisions and deliberating between options, which in turn, may have contributed to students theory-driven, rather than arbitrary trial and error approach.

\subsection{Local versus global constructions}

As expected, student-student interactions (e.g., "it looks like a cause and effect - no rain less food," "so why are some surviving and some not? That's the question") and teacher-student interactions (e.g., "why don't you look at them in a normal season?" "is there a significant difference between dead ones and live ones in their beak length or in their leg length?") in small-group discussions tended to center on choosing investigation actions and understanding specifically why the finches were dying and what enabled the surviving finches to survive. However, whole-class discussions were effective in encouraging students to reflect on what they learned from the activity and relating their understanding of the finch episode to understanding the mechanism of natural selection, as shown in the episode below.

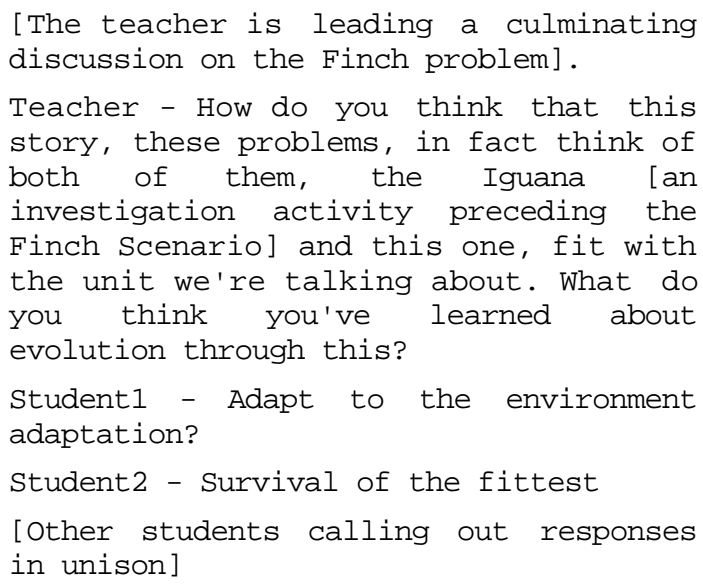

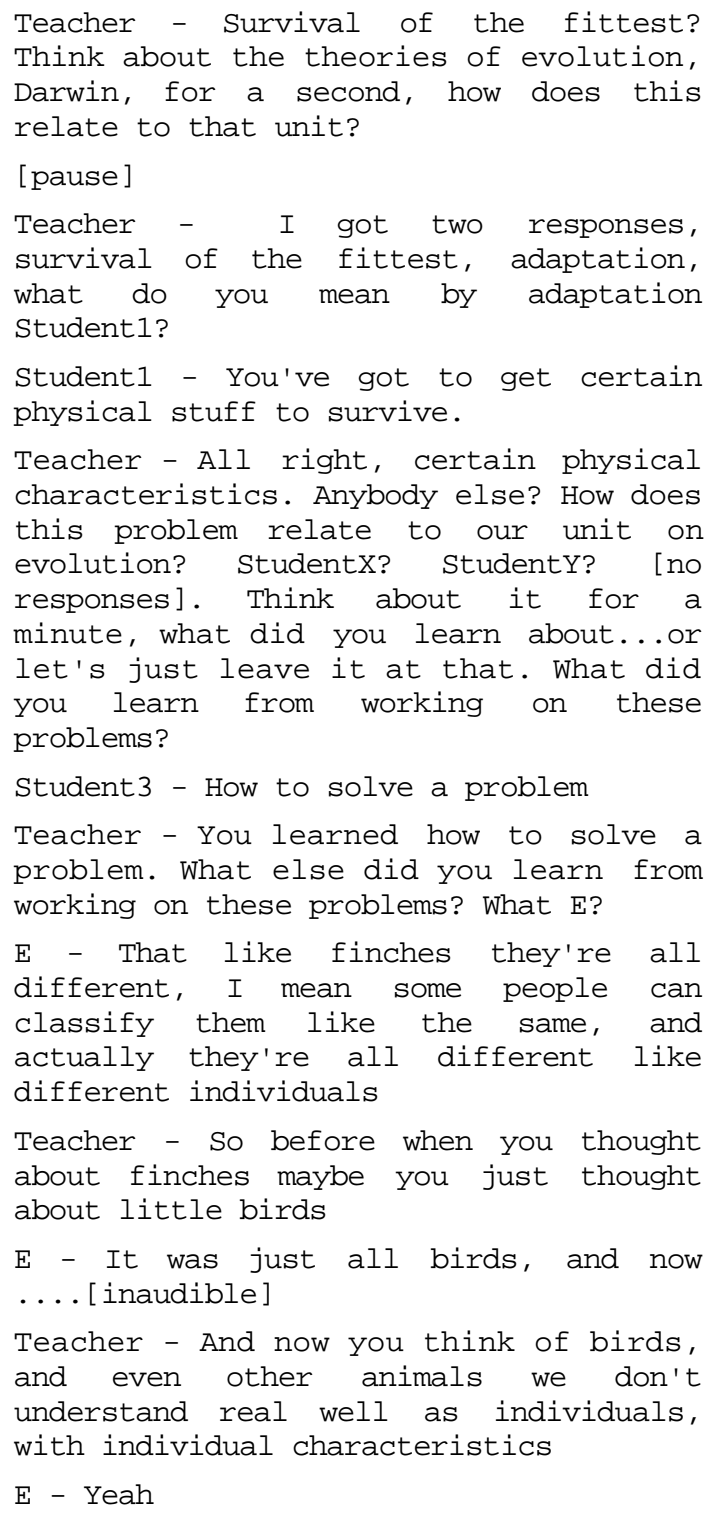

The type of discussion illustrated above may not only provide a prompt for reflection, but may also provide an opportunity for the teacher to help students move from local to global understandings by elaborating on student remarks (Lemke, 1990). Earlier research (O'Connor \& Michaels, 1993) suggests that when the teacher "revoices" student remarks in more expert terms it can help students extend their understanding and appropriate more of an expert, or scientific view. In the example above the student's response talked specifically about finches, but when the teacher revoices the student's response she replaces "finches" with "birds" and adds "other animals we don't understand real well" to reformulate the student's remark as a general principle.

Based on this discussion alone it is impossible to evaluate whether the students actually understood the terms they used and their relevance to natural 
selection. However, these discussions may be a necessary first step towards achieving this type of understanding.

\subsection{Private versus public learning opportunities}

\subsubsection{Understanding probabilistic systems}

The next two episodes demonstrate how teacherstudent interactions in small-groups during their investigations can lead to important, yet unplanned, learning opportunities that might only be experienced by a few groups unless they serve as an impetus for the teacher to introduce the same issues in a wholeclass forum which now makes these issues public and a learning opportunity for the whole class.

The first example shows how students are ready to abandon a sound theory they developed in their investigation, because they do not understand probabilistic systems (systems where only the likelihood of an outcome can be predicted, but the outcome of any particular case cannot be predicted by a law or formula). It also demonstrates the critical role that teacher intervention plays in successfully enacting science learning through student-directed inquiry. If the students had not discussed their confusion with the teacher (in this case, one of the researchers) they may have abandoned a profitable line of inquiry. Although the following exchange between the students and the researcher was not sufficient to help the students gain an understanding of probabilistic systems, it did help students realize that they are not necessarily "wrong" and convinced them to continue to pursue this line of inquiry.

Developing an understanding of probabilistic systems and the distinction between deterministic and probabilistic phenomena is a critical part of understanding science, in general, and biology in particular, since many biological systems are probabilistic. However, this is a very complex and confusing topic to understand that typically is not directly addressed by introductory high school biology curricula. The second example, shows how the teacher capitalized on the fact that this issue happened to emerge in some of the small group discussions in order to present a critical learning opportunity to the entire class. Of course, this topic is too complex to be clarified and understood through one class discussion, but once it is introduced as a topic of discussion it is more likely to be discussed in the future and considered in subsequent investigations.

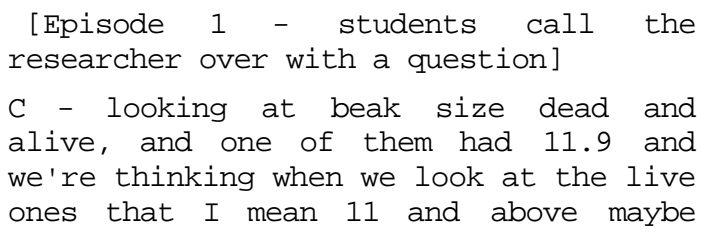

they survive, 11 and below they die, but this one he's got 11.91 and he died. So...?

Researcher - So how would you explain that? Do you think you're wrong, or do you think there's a way to explain that, that you could be right and that could still be happening?

C - I think I'm wrong. But..., but, then why did he die? Cause his is basically normal to the rest, and the leg length is 18 point something, and that's just like the other ones that survived.

Researcher - Well, is there anything just in day to day life that you know of or can think of where there's a trend or something you expect, or overall, but one or two don't really fit that?

BJ - Maybe somebody came with a big truck or something and ran over some of them...

C - Maybe, maybe [giggles]

[Episode 2 - towards the end of a culminating discussion of the Finch Scenario. Before this excerpt starts the class discussed differences between an event that randomly kills or an event that selects particular individuals where there is a pattern associated with who lives and who dies. ]

Teacher- That made me think of something that I noticed as I was watching you all work. When you were finding, when you were collecting evidence, and you started to see a story appear, and you'd pick maybe an individual profile and all of a sudden there was, you'd find a finch with a short beak that survived the dry season and what was, what was your reaction when that happened?

Student1 - [inaudible]

Teacher - They weren't part of the pattern, and so did you scrap your story and start all over again, because you found a finch that

Student2 - [inaudible]

Teacher - It was an exception? So what are you saying about your patterns, or maybe about the definition of a pattern? Does that mean that every single individual have to fall within that long beak length range for your story to hold up? Did you hold yourself to those requirements that everything had to fit exactly into your pattern? 
[pause no responses] What do you know about patterns? What do we know about normals?

Student3 - [mumbles something then retracts ]

Teacher - You learned about the bell curve in math? what does the bell curve represent?

Student3 - [inaudible]

Student 4 - There's a few at one end and a bunch in the middle.

Teacher - OK, that's a real important thing to keep in mind as a scientist, do you suppose that scientists just say "op, that's it, I've got this one piece of evidence, or this one individual characteristic that doesn't fit so I'm going to throw this theory out, this theory is no good"

\subsubsection{Constructing detailed explanations}

This example is from a whole-class discussion that served as a culminating activity for the Finch Scenario. In this discussion students and teacher, together, describe a detailed causal explanation for the episode presented in the problem. The discussion in the following excerpt has two interesting characteristics: the level of detail and extent of the causal story described, and the conversation structure. In the example the teacher responds to students by asking for extensions or continuations, rather than with evaluations. In this way she bridges student remarks and helps create a structure that is closer to student cross talk. Here students are the "talkers of science," unlike traditional question-answer-evaluation structures where the teacher is the "talker of science" (Lemke, 1990).

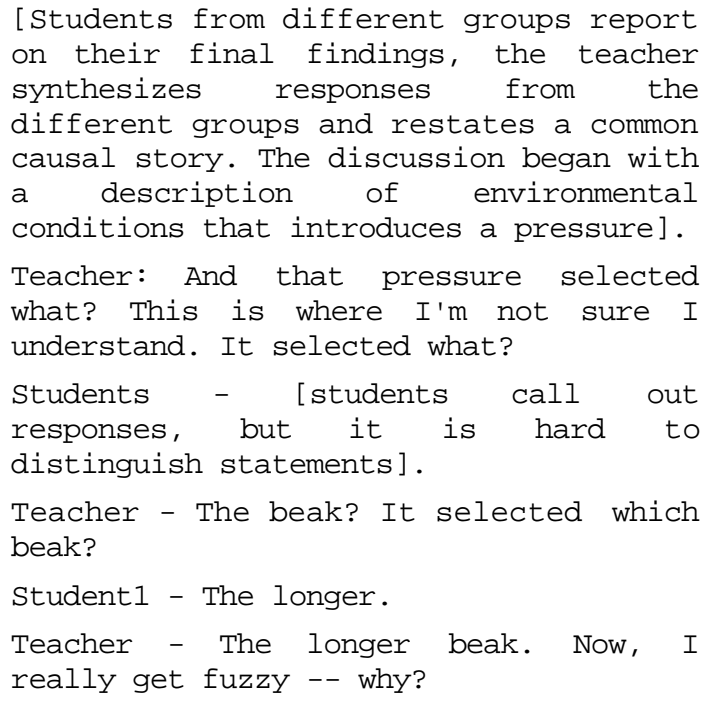

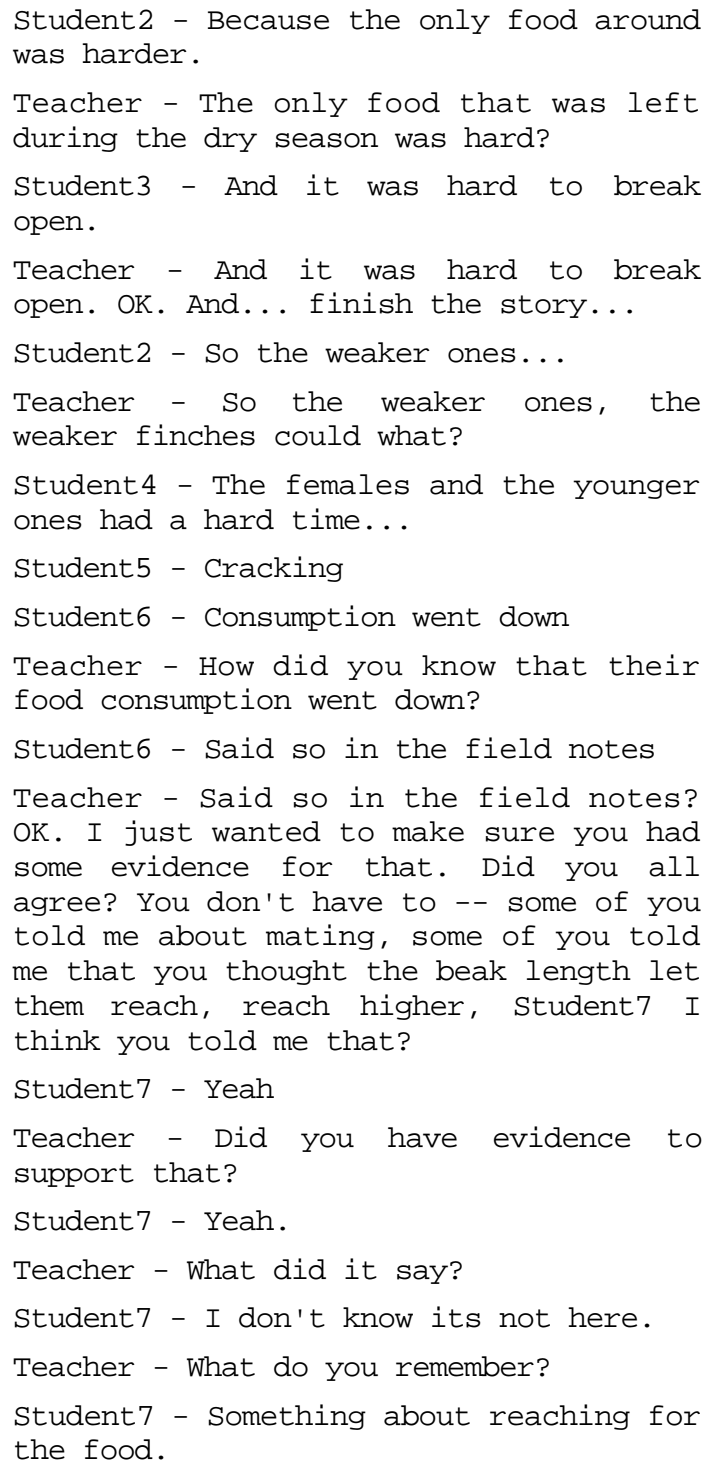

The level of detail in the explanation that was constructed during the discussion above exemplifies the advantage of whole class, cross-group discussions. Very few groups in the class constructed as complete and detailed an explanation as the one laid out in the quote above, however, this culminating discussion drew on several groups' explanations in order to construct a more complete, and detailed explanation.

\section{Conclusion}

Students learning science by collaborating on investigations can be more effective if these collaborations occur in a context that combines three types of supports. First, domain-specific strategic support focuses investigations and discussions on key principles of the domain. Second, teacher-student interactions during small-group investigations augment the support provided in the computer 
environment by modeling profitable strategies, and answering questions. Third, teacher-student interactions in whole-class forums enable the class to generalize their learning by prompting students to reflect on their experiences, and by extending the range of experiences through cross group exchanges.

Whole-class discussions that are interleaved with student-directed investigations may be particularly profitable, because the investigation experiences provide teachers and students with a common, concrete frame of reference on which to base student and teacher remarks.

We identified curricular structures that capitalize on these three types of support. There are a number of questions that need to be addressed in order to develop an instructional model for integrating computersupported student inquiry with structured classroom interaction. First, we need to determine whether students' remarks during class discussions reflect learning and understanding of the material. Second, we need to examine the range of learning resulting from discussions. Are students who take part in the discussion the only ones that appear to benefit from the discussions? Finally, we need to combine assessments of student learning with analyses of discussions in order to characterize the nature of effective and less effective discussions, and the strategies teachers employ to create these different settings.

\section{Acknowledgments}

BGuILE is supported by a grant from the James S. McDonnell Foundation. Any opinions, findings, or conclusions expressed here belong to the authors and do not necessarily reflect the views of the James S. McDonnell Foundation. We thank William Sandoval, Brian Smith and Franci Steinmuller for their collaboration on this research. We also thank David Goodspeed and Linda Patton for inviting us into their classrooms and collaborating with us as designers, and their students for their interest and cooperation.

\section{References}

Glynn, S. M., \& Duit, R. (1995). Learning Science Meaningfully: Constructing Conceptual Models. In S. M. Glynn \& R. Duit (Eds.), Learning Science in the Schools: Research Reforming Practice Mahwah, NJ: Lawrence Erlbaum Associates.
Grant, P. R. (1986). Ecology and evolution of Darwin's finches. Princeton, NJ: Princeton University Press.

Lemke, J. L. (1990). Talking science: language, learning, and values. Norwood, N.J.: Ablex.

Minstrell, J., \& Stimpson, V. (1996). A classroom environment for learning: Guiding students' reconstruction of understanding and reasoning. In L. Schauble \& R. Glaser (Eds.), Innovations in learning: New environments for education (pp. 175-202). Mahwah, NJ: Erlbaum.

O'Connor, M. C., \& Michaels, S. (1993). Aligning Academic Task and Participation Status through Revoicing: Analysis of a Classroom Discourse Strategy. Anthropology and Education Quarterly, 24(4), 318-335.

Pea, R. D. (1991). Augmenting the discourse of learning with computer-based learning environments. In E. de Corte, M. Linn, \& L. Verschaffel (Eds.), Computer-based learning environments and problem-solving (pp. 313344). New York: Springer-Verlag.

Roschelle, J. (1992). Learning by collaboration: Convergent conceptual change. The Journal of the Learning Sciences, 2, 235-276.

Roth, W.-M. (1995). Affordances of computers in teacher-student interactions: The case of Interactive Physics. Journal of Research in Science Teaching, 32, 329-347.

Shute, V. J., Glaser, R., \& Raghavan, K. (1989). Inference and discovery in an exploratory laboratory. In P. L. Ackerman, R. J. Sternberg, \& R. Glaser (Eds.), Learning and Individual Differences (pp. 279-326). New York: W. H. Freeman and Company.

Tabak, I., Smith, B. K., Sandoval, W. A., \& Reiser, B. J. (1996). Combining general and domainspecific strategic support for biological inquiry. In C. Frasson, G. Gauthier, \& A. Lesgold (Eds.), Intelligent Tutoring Systems: Third International Conference, ITS '96, (pp. 288-296). Montreal, Canada: Springer-Verlag.

White, B. Y. (1993). ThinkerTools: Causal models, conceptual change, and science education. Cognition and Instruction, 10, 1-100.

\section{Authors' Addresses}

Iris Tabak and Brian J. Reiser; School of Education and Social Policy; Northwestern University; 2115 N. Campus Drive; Evanston, IL 60208; tabak@nwu.edu; reiser@ils.nwu.edu 